\title{
Redes brasileiras de colaboração científica em Organização e Representação do Conhecimento: análise de coautorias dos Encontros Nacionais de Pesquisa em Ciência da Informação (ENANCIBs)
}

Scientific collaboration networks in Knowledge Organization and Representation: a co-authorship analysis of the Encontros Nacionais de Pesquisa em Ciência da Informação (ENANCIBs) in Brazil

\section{Ely Francina Tannuri OliveIRA (1), Maria Cláudia Cabrini GrÁcıo (2) e José Eduardo Santarém SE- GUNDO (3)}

Faculdade de Filosofia e Ciências - Unesp, - Av. Hygino Muzzi Filho, 737 Marília - São Paulo - Brasil CEP: 17525-900. (1) etannuri@flash.tv.br (2) cabrini@marilia.unesp.br (3) santarem@marilia.unesp.br

\begin{abstract}
Resumen
Se realizó un análisis de la red de colaboración científica institucional brasileña en Organización y Representación del Conocimiento a partir del estudio de las coautorías en los Encontros Nacionais de Pesquisa em Ciência da Informação (ENANCIBs) realizados en Brasil, en el periodo desde 2003 hasta 2008, a saber: en 2003, en la ciudad de Belo Horizonte, 17 trabajos; en 2005, Florianópolis, 25 trabajos; en 2006, en Marília, 23 trabajos; en 2007, en Salvador, 47 trabajos; y en São Paulo, en 2008, 19 trabajos, totalizando un corpus de 131 investigaciones. Se utilizó Pajek a fin de visualizar la red de colaboración científica entre las instituciones y SPSS para la construcción de los clusters, utilizando el método Ward y la medida de distancia euclidiana cuadrática con variables estandarizadas.
\end{abstract}

Palabras clave: Visibilidad de investigadores. Red de colaboración científica. Red de coautorías. Organización y Representación del Conocimiento. Redes sociales. Brasil. ENANCIB.

\section{Introdução}

O desenvolvimento econômico, político e social de um país está intimamente relacionado ao crescimento da ciência e tecnologia. Consequentemente, vem aumentando o interesse de especialistas e pesquisadores por estudos, avaliações e análises que permitem visualizar quanto e como as pesquisas científicas vêm crescendo, com vistas a avaliar seus resultados e traçar políticas para seu maior incremento.

\begin{abstract}
The objective of this research is to analyze the scientific contribution net in the area of Information Science, in the subject Knowledge Organization and Representation, based on the institutional coauthorships presented at the Encontros Nacionais de Pesquisa em Ciência da Informação (ENANCIBs) that happened in Brazil, from 2003 to 2008, this is: in 2003, in Belo Horizonte city, 17 works; in 2005, Florianópolis, 25 works; in 2006, Marília, 23 works; in 2007, in Salvador, 47 works; and in São Paulo, in 2008, 19 works, a total corpus of 131 researches. Pajek software was used to visualize the scientific contribution network among the institutions and SPSS for the construction of clusters, using the Ward method and measuring the Euclidean quadratic distance with standardized variables.
\end{abstract}

Keywords: Scientific contribution network. Coauthorships network. Knowledge Organization and Representation. Social networks. Brazil. ENANCIB.

A produção científica, conjunto de publicações geradas durante a realização e término das pesquisas, vem sendo cada vez mais estudada, especialmente nos últimos 40 anos, quando a explosão documentária impôs, de certa forma, a necessidade da criação de instrumentos para avaliação da ciência, particularmente de instituições de pesquisas e pesquisadores, especialmente em países, como o Brasil, onde o desenvolvimento da ciência está relacionado ao sistema de educação superior. 
A análise da produção científica de um país, de uma região ou instituição científica envolve um conjunto expressivo de indicadores bibliométricos, entre eles os indicadores de ligação. Estes são baseados na co-ocorrência de autoria, de citações e de palavras aplicadas para o mapeamento do conhecimento e construção da rede de relacionamento entre os pesquisadores, instituições e países, utilizando técnicas métricas e análises contextuais.

A análise de coautoria é medida pelo número de publicações de colaboração entre autores, sendo empregada para identificar e mapear a cooperação regional, nacional ou internacional.

A proposição desta pesquisa é analisar e construir a rede de colaboração científica através da análise da produção científica das pesquisas apresentadas em coautorias institucionais, nos ENANCIBs - Encontros Nacionais de Pesquisa em Ciência da Informação - da instituição (sociedade civil) ANCIB (Associação Nacional de Pesquisa e Pós-Graduação em Ciência da Informação), em um dos seus grupos de trabalho (GT2), denominado "Organização e Representação do Conhecimento".

\section{Objetivos}

Nesta pesquisa objetivou-se analisar a rede de colaboração científica na área de Ciência da Informação, no tema Organização e Representação do Conhecimento, a partir das coautorias institucionais nos trabalhos apresentados nos Encontros Nacionais de Pesquisa em Ciência da Informação (ENANCIBs), realizados no Brasil, no período de 2003 a 2008, entre as diferentes instituições, tanto do país, como de instituições internacionais. A partir da produção científica apresentada nos cinco últimos eventos, verificou-se, através de estudos bibliométricos, como colaboram entre si os cientistas, identificando, analisando e descrevendo a situação das redes de colaboração científica existentes.

\section{Referencial Teórico}

A ANCIB - Associação Nacional de Pesquisa e Pós-Graduação em Ciência da Informação - é uma sociedade civil, fundada em junho de 1989 , admitindo sócios institucionais (Programas de Pós-Graduação em Ciência da Informação) e sócios individuais (professores, pesquisadores, estudantes de pós-graduação e profissionais advindos dos programas). Sua finalidade é acompanhar e estimular as atividades de ensino de pós-graduação e de pesquisa em Ciência da Informação, no Brasil.
Desde sua criação, tem se projetado, no país e fora dele, como uma instância de representação científica e política, importante para o debate das questões pertinentes à área de informação.

As atividades da ANCIB estruturam-se em duas frentes: os Programas de Pós-Graduação stricto sensu, que são representados pelos seus coordenadores, e os ENANCIBs. Esses encontros constituem fórum de debates e reflexões que reúnem pesquisadores interessados em temas especializados da Ciência da Informação, organizados em Grupos de Trabalho, segundo categorias temáticas, entre eles o GT2 - Organização e Representação do Conhecimento - objeto de estudo desta pesquisa.

Os ENANCIBs acontecem desde 1994, propiciando, ao longo desses anos, edições de foro privilegiado para o debate da pesquisa conduzida pela área, agregando pesquisadores e programas de pós-graduação brasileiros, para um trabalho de prospecção e aprofundamento. Assim, através do amplo diálogo entre os pesquisadores, procura-se fazer avançar as atividades de geração de conhecimento, incentivando a reflexão sobre temas e tendências acadêmicas da atualidade, bem como contextualizando a organização da informação no universo do conhecimento, avaliando também a produção científica na área, objeto de um dos outros GTs.

O campo da Organização e Representação do Conhecimento tem como principal foco os processos de produção, tratamento, disseminação e recuperação da informação. Consequentemente, seu escopo e abrangência ultrapassam fronteiras e dialogam com outras temáticas da Ciência da Informação e de outras áreas do conhecimento.

O conceito de rede social e a análise dessas relações têm sido desenvolvidos como forma de medir a colaboração científica entre os pesquisadores, instituições e países, para visualizar a frente de pesquisa de determinada área. A análise da colaboração científica não é uma novidade do século XX, mas teve, a partir daí, seu crescimento acelerado, especialmente nas últimas décadas.

$\mathrm{Na}$ área de estudos métricos, especialmente no tema "redes de colaboração científica", destacase a Espanha, com estudos liderados por Liberatore, Herrero-Solana, Moya-Anégon, Olmeda Gómez e Molina, entre outros. Ainda nos E.U.A: Garfield, Price, Katz e Frame; no Reino Unido: Meadows e Thelwall; na Bélgica: Rousseau e Glänzel. Estes pesquisadores têm se destacado no tema, nos diferentes países citados. 
O pesquisador Smith, em 1958, citado por Balancieri et al (2005, p. 3), foi um dos primeiros pesquisadores a observar o crescimento da publicação em coautoria e a sugerir o seu uso como uma medida de colaboração entre grupos de pesquisadores ou instituições de um mesmo país ou colaboração internacional.

$\mathrm{Na}$ década de 1960, destaca-se o início dos estudos na área da colaboração científica. Entre as constatações nesse período, observou-se que a colaboração científica se inicia nas relações entre orientador e orientando e, especialmente, no âmbito dos "colégios invisíveis".

Assim, a colaboração científica é um dos fenômenos mais visíveis observados na construção da ciência ao longo da história. Price (1963), citado por Barragan et al. (2006, p. 414), foi um dos primeiros pesquisadores a apontar evidências empíricas do aumento das coautorias na ciência.

Segundo Balancieri et al (2005, p. 2), "a colaboração científica oferece uma fonte de apoio para melhorar o resultado e maximizar o potencial da produção científica".

Segundo Wasserman e Faust (1994, p. 9), "o termo 'rede social' se refere ao conjunto de atores e suas ligações entre eles". A análise de rede tem por objetivo modelar as relações entre os atores, a fim de retratar, descrever e representar a estrutura de um grupo, quer seja composto por países, instituições ou pessoas.

O conceito de análise de rede social desenvolveu-se a partir de uma confluência da teoria social com a metodologia matemática, estatística e computacional.

O importante ganho resultante das autorias múltiplas, sejam duplas, triplas ou $n$-uplas, quando comparado ao trabalho de pesquisadores isolados, é a ampliação do repertório de abordagens e ferramentas, que advém do intercâmbio de informações e da produtividade que se verifica quando grupos, pesquisadores ou instituições distintas juntam esforços no sentido de determinada meta, promovendo a interação entre os pesquisadores.

A colaboração científica entre autores ou instituições (Olmeda Gómez, Perianez-Rodriguez e Ovalle-Perandones, 2008) supõe um compartiIhamento de ideais centrais de um projeto, os objetivos e as consequências que geraram essas idéias: é necessário um clima de confiança, o estabelecimento de uma divisão de trabalho e interação entre os investigadores, compartilhamento de informações e coordenação destas diferentes relações do investimento conjunto, e redação final. Porém, o mais relevante é que muitas vezes a coautoria, além de significar investimento conjunto, é uma oportunidade para troca do conhecimento "tácito", do conhecimento novo e atual, com que se passa a ter maior autoridade do ponto de vista epistemológico, pondo assim em evidência a resolução de problemas e questões comuns. A coautoria reflete todo o rol possível de intercâmbios e trocas entre os pesquisadores, como são, por exemplo, as conversas informais e as discussões em congressos e palestras advindas das apresentações de pesquisas.

\section{Metodologia}

O universo de pesquisa foi constituído pela produção científica apresentada no GT2, Organização e Representação do Conhecimento, nos cinco últimos Encontros (período de 2003 a 2008), totalizando 131 trabalhos de pesquisa, entre as comunicações orais e pôsteres, assim distribuídos:

- 17 trabalhos de pesquisa apresentados no $\mathrm{V}$ ENANCIB, realizado em Belo Horizonte (MG), em 2003;

- 25 trabalhos de pesquisa apresentados no VI ENANCIB, realizado em Florianópolis (SC), em 2005;

- 23 trabalhos de pesquisa apresentados no VII ENANCIB, realizado em Marília (SP), em 2006;

- 47 trabalhos de pesquisa apresentados no VIII ENANCIB, realizado em Salvador (BA), em 2007;

- 19 trabalhos apresentados no IX ENANCIB, realizado em São Paulo (SP), em 2008.

O objeto de estudo foi constituído desses 131 trabalhos de pesquisas, da fonte já citada.

Justifica-se o recorte temporal feito nos últimos cinco ENANCIBs por se considerar a quantidade de trabalhos representativa para o estudo em questão, além de que, somente a partir de 2003, os trabalhos foram disponibilizados on-line.

Considerando o caráter instrumental desta pesquisa, a abordagem usada foi quantitativa e qualitativa, inicialmente descritiva, como também exploratória e analítica. Em um primeiro momento, realizou-se a leitura das 131 pesquisas, todas referentes à Organização e Representação do Conhecimento, dentro de suas diferentes abordagens (Formação profissional, Atuação profissional, Produção científica, Impacto tecnológico), quer sejam: Leitura Documentária, Identificação e Seleção de Conceitos, Condensação Documentária, Representação 
Documentária, Recuperação da Informação, Resumos e Índices (Produtos da organização da informação), Sistemas de Classificação, Cabeçalhos de Assunto, Tesauros, Ontologias e Terminologias (Instrumentos de organização da informação), Lógica, Linguística, Terminologia, Diplomática, Psicologia Cognitiva, Tecnologias de Informação e Comunicação (que constituem a interdisciplinaridade).

Foi construída uma base de dados, utilizando o Microsoft Access e as variáveis em estudo organizadas em tabelas e reconstruídas no software Excel.

A partir da leitura dos trabalhos, levantaram-se aqueles em autoria simples e múltipla, resultando em 42 trabalhos com autoria simples ( $32 \%)$ e 89 em coautorias ( 68\%), entre as duplas, triplas e $n$-uplas. Calculado o índice de colaboração (Spinak, 1996) dos trabalhos, encontrouse 2,01 , que indica a média de autores por trabalho.

Do grupo de 89 trabalhos em coautoria, verificou-se quais as instituições de origem. Os trabalhos em coautorias foram advindos de 41 instituições, em âmbito nacional e internacional.

Foi construída uma matriz quadrada e simétrica, $41 \times 41$, a partir da frequência de coautorias entre essas instituições, para posterior construção da rede de colaboração.

Ressalte-se que as instituições, apesar de aparentemente e nominalmente terem a mesma origem, foram apresentadas separadamente, por serem de cidades e regiões distintas, e com contextos sociais, filosóficos e, possivelmente, epistemológicos diferentes.

Foi utilizado o software Pajek a fim de visualizar a rede de colaboração científica entre as instituições. Para a representação da rede de colaboração institucional utilizou-se, no menu layout do Pajek, a opção Energy/Kamada-Kawai/Separate components.

Utilizou-se o SPSS (Statistical Package for de Social Science) para construção dos clusters, usando o método Ward e medida de distância euclidiana quadrática com variáveis padronizadas.

Após a construção da rede de colaboração e do dendograma, destacaram-se as temáticas trabaIhadas em colaboração na sub-rede mais densa, que compuseram também um dos clusters, através das palavras-chave encontradas em comum, nas pesquisas deste grupo em destaque.

\section{Apresentação e análise dos dados}

Grande número (64) dos trabalhos foi apresentado em autoria dupla e 16, em autoria tripla. Desse total de 80 trabalhos, 49 deles, ou seja, $61 \%$ apresentaram-se orientando e orientador do mesmo programa de Pós-Graduação. Temse, por hipótese, que este panorama é reflexo do caráter do próprio evento, onde orientando e orientador da mesma instituição apresentam trabalhos em coautorias, além de ratificar a constatação dos pesquisadores relativa à questão da história da colaboração científica.

Apresenta-se, a seguir, a rede de coautorias, construída com as 41 instituições autoras dos trabalhados em colaboração. Esclarece-se que as áreas dos círculos se referem à freqüência de coautorias dentro da própria instituição, a espessura dos segmentos de reta, a intensidade da colaboração institucional. Os círculos vermeIhos representam as instituições com colaboração interna e os em branco, sem coautoria interna.

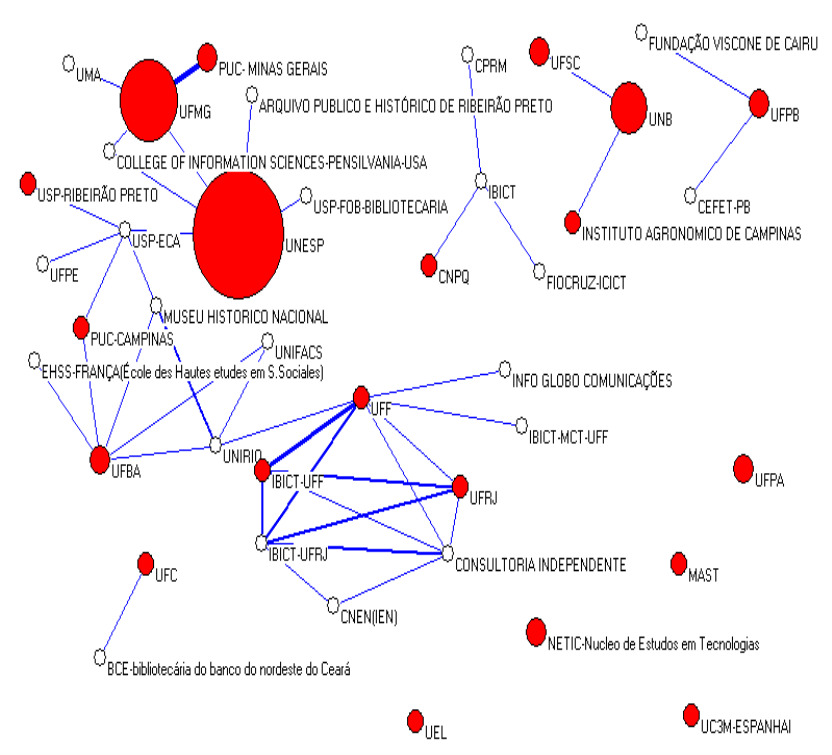

Figura 1. Rede de colaboração científica

$\mathrm{Na}$ análise da rede, destaca-se o principal componente da rede composto por 23 instituições, que se apresenta com três sub-redes mais significativas, a saber: a primeira com núcleo na Unesp (com alta coautoria interna), constituída pelas seguintes instituições: UFMG, USP (FOB), USP (ECA), Arquivo Público de Ribeirão Preto, Collage Of Information Science (E.U.A.), UMA e PUC-MG. Observa-se que a conexão mais forte entre instituições encontra-se nesta sub-rede entre UFMG e PUC-MG. 
A segunda sub-rede apresenta núcleo na UFBA e conexão com a primeira sub-rede através da USP-ECA. Suas conexões não apresentam proximidade geográfica, destacando-se que a mesma se conecta com a terceira sub-rede através da UNIRIO. Esta sub-rede, com núcleo na UFF, UFRJ e IBICT/UFF, é a mais densa, além de ter, geograficamente, instituições mais próximas.

Ainda na rede observam-se quatro outros componentes (sub-redes) menos significativos $\mathrm{e}$ mais isolados, centrados no IBICT, na UNB, na UFPB e na UFC, além de cinco instituições que só apresentaram colaboração interna.

Calculou-se a densidade da rede de colaboração, tomando-se o quociente entre o número de conexões presentes na rede (46) pelo número total de conexões possíveis (820), resultando em 0,056 , configurando $5,6 \%$ das possibilidades de conexões, indicador que sugere uma frágil relação entre as instituições.

A rede de colaboração se apresenta, portanto, por um lado, com algumas instituições isoladas, e por outro, formando a configuração de uma rede científica, porém pouca densa.

Vale lembrar que as posições em destaque não são características das próprias instituições, mas refletem aspectos relacionais desta rede em particular, gerada a partir de um evento significativo da área, em um período de tempo determinado.

Em seguida, apresentam-se os clusters, visualizados através do dendograma.

$\mathrm{Na}$ leitura do dendograma, destacam-se três grupos constituídos, segundo padrão de similaridade de colaboração institucional. O primeiro e maior, constituído por 34 instituições, iniciandose com o CPRM até a USP-ECA, pode ser caracterizado pela pouca colaboração interna $\mathrm{e}$ modesta colaboração interinstitucional. $O$ segundo grupo, composto pela Unesp e UFMG, possui alta colaboração intra-institucional e coautoria com várias outras instituições, porém de forma pulverizada, no entanto, sem interlocução entre as mesmas. O terceiro grupo é composto por instituições do Rio de Janeiro que se caracterizam por alta colaboração científica interinstituição, determinando uma densa e forte parceria entre todas elas, com baixa colaboração intrainstitucional.

O terceiro cluster constitui o core da sub-rede mais densa, formada por instituições do Estado do Rio de Janeiro. Tem como principal característica pesquisas sobre as seguintes temáticas: Ontologia; Teoria da Classificação, Mapas conceituais e Taxonomia; Indexação, Tesauros e
Terminologia; Mecanismos de busca e Recuperação da Informação. Observe-se ainda a proximidade geográfica, como um possível agente facilitador da interlocução entre essas instituições.

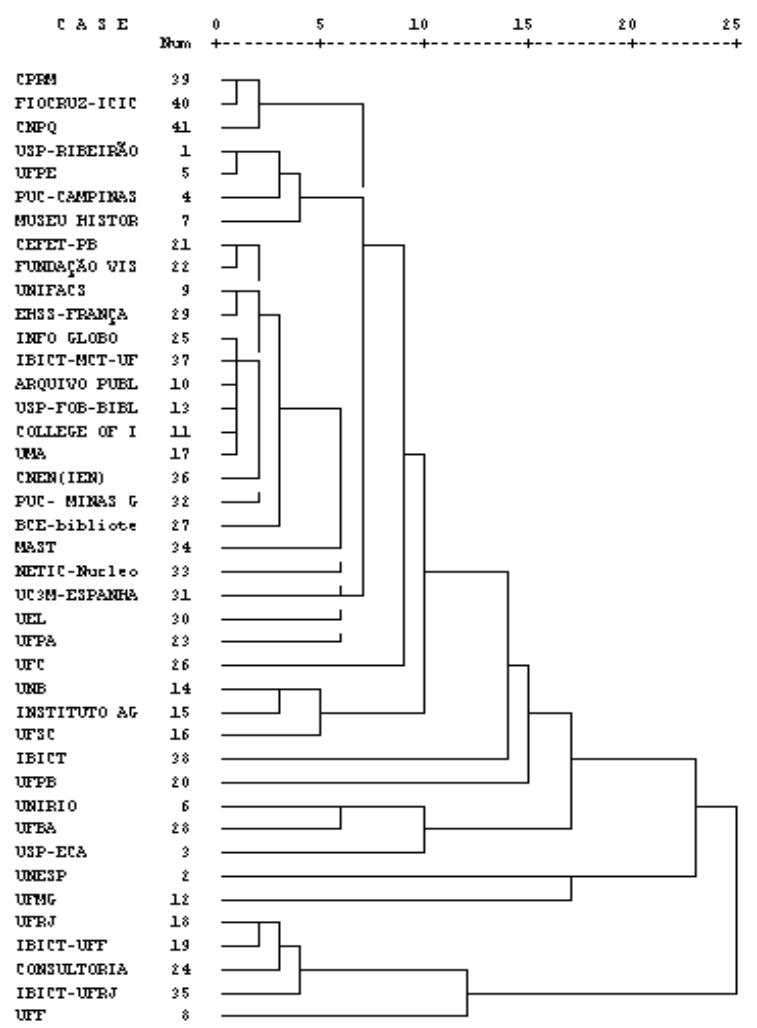

Figura 2. Dendograma gerado a partir da matriz de coautorias

\section{Considerações finais e recomendações}

Algumas instituições tendem a trabalhar de forma isolada, com muitas coautorias intragrupo institucional, porém as colaborações entre instituições, apesar de existentes, acontecem em menor número.

Os resultados apontam e recomendam outros possíveis trabalhos e investigações, em particular, a comparação com as redes determinadas por outros eventos e instituições, por periódicos indexados pelo ISI no mesmo tema, bem como a rede formada por pesquisadores de outros GTs do mesmo evento, e ainda a frente de pesquisa formada pela análise de citação e cocitação nestes GTs.

\section{Referências}

Balancieri, Renato; Bovo, Alessandro Botelho; Kern, Vinicius Medina; Pacheco, Roberto Carlos dos Santos; Barcia, Ricardo Miranda (2005). A análise de redes de colabo- 
ração científica sob as novas tecnologias da informação e comunicação: um estudo na Plataforma Lattes. // Ciência da Informação. 34:1 (jan./abr. 2005) 64-77.

Barragan, Maria J., Guerrero-Bote, Vicente P., MoyaAnégon, Félix (2006). Colaboración Científica de España com América Latina y el Caribe. // Anales del VII Encuentro Asociación de Educadores e Investigadores de Bibliotecología, Archivología, Ciencias de la Información y Documentación de Iberoamérica y el Caribe, Marília, SP, nov., 2006. http://www.edibcic.org/VII_EDIBCICl VIIEDIBCIC.htm.

Cervantes, Brigida M. Nogueira; Euclides, Maria Luzinete; Xavier, Raphael Figueiredo; Herrero-Solana, Victor (2006). Análise das redes de colaboração científica em Ciência da Informação: uma experiência Brasileira com o currículo Lattes/CNPq. // Anales del VII Encuentro Asociación de Educadores e Investigadores de Bibliotecología, Archivología, Ciencias de la Información y Documentación de Iberoamérica y el Caribe, Marília, SP, nov., 2006. http://www.edibcic.org/VII_EDIBCIC/VII EDIBCIC.htm.

Filippo, Daniela de; Casado, Elías Sanz; Gómez, Isabel (2007). Movilidad de investigadores y producción en coautoria para el estudio de la colaboración científica. // Revista CTS. 3:8 (abril 2007) 23-40.

Frame, Davidson; Carpenter, Marck (1979). International Research Collaboration. // Social Studies of Science. 9 (1979) 481-497.

Liberatore, Gustavo; Herrero-Solana, Victor; Guimarães, José Augusto Chaves (2007). Análise bibliométrica do periódico brasileiro Ciência da Informação durante o período 2000-2004. // Brazilian Journal of Information Science, 1:2 (jul./dez. 2007) 3-21. http://www.bjis. unesp.br/pt/.

Mingoti, Sueli Aparecida (2007). Análise de dados através de métodos de estatística multivariada: uma abordagem aplicada. Belo Horizonte: Editora UFGM, 2007.

Molina, José Luis; Muñoz, Juan Manuel; Domenech, Miquel (2002). Redes de publicaciones científicas: un análisis de la estructura de coautorias. // Redes: Revista Hispánica para el Análisis de Redes Sociales. 1 (2002). http://revista-redes.rediris.es/pdf-vol1/vol1_3.pdf (24 de outubro de 2008).

Ohira, Maria Lourdes Blatt (1997). Produção técno-científica e artística da Universidade do Estado de Santa Catarina (1991-1995). // Witter, Geraldina Porto (org.). Produção científica. Campinas: Editora Átomo, 1997. 87-113.

Olmeda Gómez, Carlos; Perianez- Rodriguez, Antonio; Ovalle-Perandones, Maria Antonia (2008). Estructura de las redes de colaboración científica entre las universidades españolas. // Ibersid. (2008) 129-140.

Spinak, Ernesto (1996). Dicionario enciclopédico de Bibliometria, Cienciometria e Informetria. Venezuela: UNESCO, 1996.

Wasserman, Stanley; Faust, Katherine (1994). Social network analysis: methods and applications. Cambridge: Cambridge University Press, 1994. 